\title{
Pathways to school belonging
}

School belonging is generally regarded as a student's sense of affiliation or connection to his or her school. Anyone who has personally navigated the sometimes torturous terrain of secondary school is able to have some level of direct understanding of the importance that belonging, fitting in, and identifying with a school holds for most people. Educators and practitioners often work with young people who feel that they do not belong to the school community, in which they attend. An absence of belonging can manifest itself in mental health concerns, school attrition, and risk taking behaviours. Opportunities for early intervention through fostering school belonging are born from a greater understanding and awareness of what school belonging is and how it is contextualised and fostered. This special issue aims to place a focus on school belonging and highlight it as a significant social issue of our time.

A powerful impetus for this special issue was to create a resource which offers a high level of applied impact for both researchers and practitioners. This is evident in the high quality and variance in the collection of articles that are presented in this issue. The first paper of this special issue by Slaten, Ferguson, Allen, Vella-Brodick and Waters, School belonging: A review of the history, current trends, and future directions, provides an overview of school belonging through a review of literature that describes the current context, trends and relevancy for future research. Most notable in this article is a discussion of school belonging in the university context. Given that the overarching school belonging literature is mainly concerned with issues in primary and secondary schools, this article is unique in exploring new ground in tertiary settings, where there is a dearth of academic research.

The second article of this special issue, by Furlong, Moffa, and Dowdy, provides further insight into the application of school belonging in school settings by examining the construct's value in mental health screening for psychological distress and life satisfaction. In their article, Exploring the contributions of school belonging to complete mental health screening, the authors found that students who reported high levels of life satisfaction and normative distress ("thriving") reported a higher sense of belonging than students who experienced low life satisfaction and elevated distress ("troubled"). In the second part of their analysis, they found that school belonging served as a predictor for social and emotional wellbeing one year on, but offered very little explained variance towards psychological distress symptoms. The authors argue that, although school belonging did not contribute substantially to psychological distress, it still has an important place in the complete mental health screening of secondary school students.

The special issue is particularly interested in considering school belonging in a range of populations. This is exemplified by Due, Riggs and Augoustinos, who used a novel methodology of photographic elicitation techniques in their paper, 
Experiences of school belonging for young children with refugee backgrounds. The authors found that students with refugee backgrounds were able to derive a sense of school belonging from their environment, which included their relationships with peers and teachers. The authors offer suggestions for schools catering for children with refugee backgrounds.

Literature on school belonging often focuses on student experiences and perspectives. Gowing and Jackson rigorously extend the literature by drawing on school staff as well. In Connecting to School: Exploring student and staff understandings of connectedness to school and the factors associated with this process, the authors contextualise school connectedness, "as a process rather than a state, fluctuating across time within the relational, experiential, and physical spaces of school life" (p. 54). The article highlights the importance of the teacher and peer relationship for school belonging, but also presents seminal findings in relation to joint decision making between young people and parents on choice of school and distance of home to school, which may facilitate greater opportunities for extra-curricular activities.

Coker, Martinez, McMahon, Cohen and Thapa extend our understanding of extracurricular activities in their paper, Involvement in extracurricular activities: Identifying differences in perceptions of school climate. A central finding of their work is that extracurricular activities are beneficial for school connectedness, which affords readers a greater understanding of the role of this predictor in school belonging. The authors examine how different extra-curricular activities (sports, clubs and the arts) interact with school connectedness and find that greater involvement in extra-curricular activities does not necessarily equate to higher school connectedness. In fact, the types of extra-curricular activities and the way they combine play a fundamental role in a young person's sense of belonging.

One of the strengths of this issue is in presenting research on school belonging that has used a range of methodologies. Another path to belonging: A case study of middle school students' perspectives, by Green, Emery, Sanders and Anderman, shows again the value of qualitative research in this important area. The authors make an important distinction between social belonging and academic belonging and their findings elucidate both of these types and the factors that help and hinder these two constructs through the perspectives of young people at school.

The final paper of the special issue aims to distil the research on school belonging and re-frame it into an applied practical format that can be used by school leaders and practitioners. In their paper, Fostering school belonging in secondary schools using a socio-ecological framework, Allen, Vella-Brodrick and Waters present a socio-ecological framework for schools. The authors argue that school leaders and educators should be encouraged to foster students' sense of belonging by building qualities within the students and by changing school systems and processes. The framework represents the importance of whole school approaches by discussing the role of governmental, organisational, relational and individual level variables in influencing school belonging.

School belonging is a vitally important psychological construct. Taken together, the findings of the studies featured in this special issue on school belonging have relevance for intervention design and organisational structures within educational settings, especially in respect to policy and practice. The school management, in particular, have an important role in building school belonging for individuals and 
ensuring that this concept is prioritised as a guiding principle in education. The applied practice outcomes derived from this special issue will help create stronger school communities and contribute to the practice and science of educational and developmental psychology.

Kelly Allen, PhD

Guest Editor

Chris Boyle, $\mathrm{PhD}$

Editor 